\title{
New Multimode Sonotrodes Models Designed for Rotary Ultrasonic Welding Systems
}

\author{
P. Kogut*, A. Milewski, W. Kardyś, P. Kluk and P. Gawryś \\ Tele and Radio Research Institute, Ratuszowa 11, 03-450 Warszawa, Poland
}

\begin{abstract}
The paper presents methods of designing sonotrodes, working in varied mechanical vibration modes in high power application regime such as rotary ultrasonic welding systems. The aim of the sonotrode design process is to optimize its shape to achieve the best energy efficiency for a given welding process. Sonotrode geometry influences the power transferred to the welded element because it determines the vibration amplitude distribution in the sonotrode. The most popular applications use sonotrodes designed for axial vibration. Conducted research have shown that the optimal displacement and strain distribution is achieved for sonotrodes working in various vibration modes such as axial, transverse, radial or their compositions. In the paper examples of multimode sonotrode models for various rotary ultrasonic welding technologies have been presented.
\end{abstract}

DOI: 10.12693 /APhysPolA.124.474

PACS: 43.40.-r, 43.40.+s, 43.35.+d, 43.38.+n, 43.38.-p, 46.40.-f

\section{Introduction}

Ultrasonic welding is a joining technique, where ultrasonic acoustic vibrations are transferred in materials to be joined being held together under pressure (see Fig. 1). Mechanical vibrations are generated by the ultrasonic stack which is composed of a converter, a booster (if used) and a sonotrode (see Fig. 2). Converter is a device that transforms electrical energy into mechanical vibrations. In ultrasonic power applications piezoelectric sandwich transducers are used as a converter (see Fig. 2). Sandwich transducers are designed as half wave resonators of axial vibrations, tuned to resonate in ultrasonic frequency range (typically $20,30,35$, or $40 \mathrm{kHz}$ ). The ultrasonic transducer is mounted on the top surface of the sonotrode as has been shown in Fig. 2. Sonotrode and booster are the mechanical waveguide resonators tuned to converter resonant frequency. Booster and sonotrode are also mechanical transformers of mechanical force and wave speed. Mechanical vibrations in booster and sonotrode are constrained by the converter, at the input surface A1 (see Fig. 2). Booster is a half wave resonator of axial vibrations, applied (between converter and sonotrode) to boost up the mechanical axial displacement amplitude. The sonotrode transfers mechanical energy to the welded elements. Energy is transferred by the sonotrode to the junction surface (interfaced to the workpieces), which is called working surface.

In rotary ultrasonic welding systems joined materials are rolled between a rotational sonotrode and an anvil which have been presented in Fig. 1a and b. Working surface of the rotary sonotrode is placed on the perimeter of the bottom (output) surface of the sonotrode (see Figs. 1 and 2). The rolling of the material permits the

\footnotetext{
*corresponding author; e-mail: pawel.kogut@itr.org.pl
}
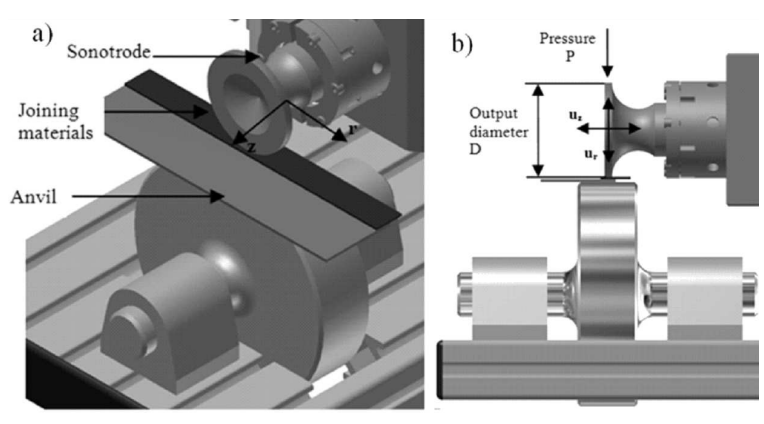

Fig. 1. Rotary ultrasonic welding system, $u-$ mechanical displacement, $u_{z}$ - axial, $u_{\mathrm{r}}$ - radial.

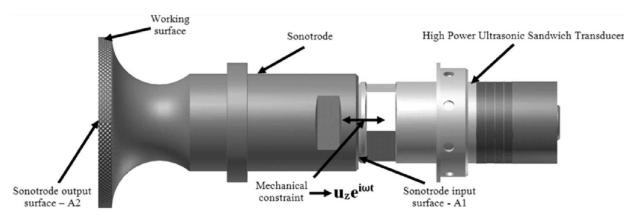

Fig. 2. High power ultrasonic vibration system.

welding process to be continuous.

Continuous welding due to stationary welding has many advantages such as:

- Higher processing speed;

- Lack of the seam gaps or overlaps.

Those advantages contribute to the growing popularity of rotary ultrasonic welding technology. In reference to growing market interest and increased demand for that technology more efficient solutions are needed. One of the most essential element in rotary ultrasonic system is the sonotrode because it transfers mechanical energy to the workpieces. Proper design of the sonotrode ensures good parameters of the weld and welding process efficiency. 


\section{Sonotrode design methods}

Mechanical vibration behavior of the waveguide resonator is described by the equation of motion which can be noted (using the Einstein notation convention) as

$$
T_{j i, j}+\rho f_{i}=\rho \ddot{u}_{i},
$$

where $\boldsymbol{T}$ - stress tensor, $\boldsymbol{f}$ - external forces, $\rho$ - material density, $\boldsymbol{u}$ - displacement vector. In rotary ultrasonic welding axially isotropic circular shaped resonators are used. Taking under consideration the axial symmetry of the sonotrode and assuming there are no energy losses and no interaction with external forces Eq. (1) can be simplified to a well known Helmholtz horn equation which can be written in the cylindrical coordinates as

$$
\frac{\partial^{2} u_{\mathrm{r}}}{\partial r^{2}}+\frac{1}{r} \frac{\partial u_{\mathrm{r}}}{\partial r}+\left(\frac{\partial^{2}}{\partial z^{2}}+k^{2}\right) u_{z}=0
$$

where $u_{\mathrm{r}}$ - radial displacement, $u_{z}$ - axial displacement, $k$ - wave vector. Assuming propagation of the plane waves in the axial direction the Helmholtz equation can be reduced to the also well known Webster horn equation

$$
\frac{\partial^{2} u_{z}}{\partial z^{2}}+\frac{1}{A} \frac{\partial A}{\partial z} \frac{\partial u_{z}}{\partial z}+k^{2} u_{z}=0
$$

where $A-$ is a cross-section along $z$ axis. Solving Eqs. (2) and (3), formulae for mechanical displacement and stress distribution can be obtained. Equation (3) can only be applied when axial wavelength is much greater than radial dimensions of the resonator. Equations (2) and (3) can be analytically solved for many interesting geometry shapes such as cylindrical, exponential, conical, and others [1-5]. Equation (2) gives more accurate results than (3) however it is more difficult to carry out the exact solution. Most known solutions of Eq. (2) are derived with approximation or semi-analytical methods $[4,5]$.

For cases where analytical solutions cannot be applied or are hard to carry out numerical methods can be used such as the finite element method (FEM). The basic assumption of this method is to approximate given geometry by the easier geometries creating sočalled mesh. The mesh consists of nodes which are interpolation points linearly connected together. Mechanical behavior of each node point is described by the proper equation of motion. The whole mechanical behavior FEM model can be obtained by solving the following algebraic equation of motion:

$$
[M]\{\ddot{u}\}+[K]\{u\}=0,
$$

where $[M]$ is mass matrix, $\{u\}-$ mechanical displacement vector, $[K]$ - stiffness matrix. Applying proper boundary conditions Eq. (4) can be solved for whole resonator system. More information about FEM can be found in [6].

\section{Acoustic power and optimization}

The most important factors in rotary ultrasonic welding process are welding speed and efficiency. Welding speed pertains to maximal materials length that can be joined in a time unit (mostly given in $\mathrm{m} / \mathrm{min}$ ), which can be noted as

$$
v_{\mathrm{w}}=\frac{l_{\mathrm{w}}}{t_{\mathrm{w}}}
$$

where $v_{\mathrm{w}}$ - welding speed, $l_{\mathrm{w}}$ - welded material length, $t_{\mathrm{w}}$ - welding time. Welding speed and efficiency depend on the acoustic power $P_{\mathrm{ac}}$ that can be transferred to the elements that are to be joined and linear rolling speed of the sonotrode. For every type of the bonding material there is a characteristic energy $E_{\mathrm{w}}$ that is needed to weld the materials. Higher acoustic power decreases welding time by supplying energy $E_{\mathrm{w}}$ in shorter time which can be noted as

$$
E_{\mathrm{w}}=P_{\mathrm{ac}} t_{\mathrm{w}} \rightarrow t_{\mathrm{w}}=\frac{E_{\mathrm{w}}}{P_{\mathrm{ac}}} .
$$

Welding speed is limited by linear sonotrode rolling speed $v_{\mathrm{s}}$, which cannot be too high to ensure welding

$$
v_{\mathrm{w}} \geq v_{\mathrm{s}}=\omega_{\mathrm{s}} r_{\mathrm{s}},
$$

where $\omega_{\mathrm{s}}$ - angular speed of the sonotrode, $r_{\mathrm{s}}$ - radius of the output surface of the sonotrode (see Fig. 2). Summing up and concluding maximal material length that can be welded is equal to

$$
v_{\mathrm{w}}=l_{\mathrm{w}} \frac{P_{\mathrm{ac}}}{E_{\mathrm{w}}} \geq \omega_{\mathrm{s}} r_{\mathrm{s}} \rightarrow l_{w_{-} \max }=\sup \left(\frac{E_{\mathrm{w}} \omega_{\mathrm{s}} r_{\mathrm{s}}}{P_{\mathrm{ac}}}\right) .
$$

Acoustic power $P_{\mathrm{ac}}$ and radius $r_{\mathrm{s}}$ (or diameter $D$ ) depends on the geometry of the sonotrode, whereas angular speed $\omega_{\mathrm{s}}$ and energy $E_{\mathrm{w}}$ is independent of sonotrode properties. Equation (8) leads to the following conclusions:

- Treating $P_{\mathrm{ac}}$ as constant value, radius $r_{\mathrm{s}}$ should be as high as possible to achieve the best performance,

- The higher $P_{\mathrm{ac}}$ the higher maximal linear speed of the sonotrode (and as well welding process performance),

- $P_{\mathrm{ac}}$ and radius $r_{\mathrm{s}}$ need to be optimized.

Assuming that only plane waves are transferred to workpieces, transferred acoustic power can be noted as

$$
P_{\mathrm{ac}}=\oint_{A} \boldsymbol{I}_{\mathrm{av}} \cdot \mathrm{d} \boldsymbol{A}=\frac{1}{2} \oint_{A} \frac{p^{2}}{\rho c} \boldsymbol{n} \cdot \mathrm{d} \boldsymbol{A},
$$

where $\boldsymbol{I}_{\mathrm{av}}$ - average sound intensity, $A$ - working surface area, $p$ - acoustic pressure, $\rho$ - medium density, $c$ - sound speed, $n-$ sound wave surface normal. To simplify the calculations the following assumptions were made:

- Plane wave vector is parallel to normal vector of surface $A \rightarrow \boldsymbol{n} \cdot \mathrm{d} \boldsymbol{A}=n \mathrm{~d} A$,

- Joined materials are so thin that their influences on the sound propagation can be neglected. Sound waves propagate directly from sonotrode to anvil with no reflection,

- Acoustic pressure is equal to normal stress $T_{\mathrm{nn}}$, 
- Mechanical displacement in direction $n, u_{\mathrm{n}}$ is equal to

$$
u_{\mathrm{n}}=A \mathrm{e}^{\mathrm{i} k n}+B \mathrm{e}^{-\mathrm{i} k n}=A \mathrm{e}^{\mathrm{i} k n} .
$$

Taking under consideration those assumptions acoustic power can be noted as

$$
\begin{aligned}
P_{\mathrm{ac}} & =\frac{1}{2} \frac{T_{\mathrm{nn}}^{2}}{\rho c} A=\frac{1}{2} \frac{\left(\sigma_{\mathrm{nn}} u_{\mathrm{n}, \mathrm{n}}\right)^{2}}{\rho c} A=\frac{1}{2} \frac{\left(\mathrm{i} \rho c^{2} k u_{\mathrm{n}}\right)^{2}}{\rho c} A \\
& =-\frac{1}{2} \rho c \omega^{2} u_{\mathrm{n}}^{2} A,
\end{aligned}
$$

where $\sigma_{\mathrm{nn}}$ - normal strain tensor, $k$ - wave vector, $\omega$ - angular frequency.

In real welding conditions mechanical vibrations are not only parallel directed and uniformly distributed to welded material surface. For different bonding materials there are different types of mechanical constraints that give better results. For example, thermoplastic elements are better welded when mechanical vibrations are parallelly directed to welded material surface (Eq. (10)), whereas for metal bonding they need to be perpendicularly directed. To improve welding performance working surface is often covered by knurl of different shapes. Knurling surface results in higher friction on working surface and constraints other vibration modes in joining materials. Treating Eq. (10) as first approximation of the transferred energy, it occurs that energy depends on the mechanical displacement distribution.

Summarizing the optimization process should lead to such sonotrode geometry in which the highest displacement amplitude, largest diameter $D$, and minimal stress amplitude are achieved. To obtain the best efficiency, the distribution of the mechanical displacement should be concentrated on the working surface.

\section{Proposed models}

The main subject of investigations described in this paragraph was optimization of the sonotrodes geometry, which improves the efficiency of welding. Proposed sonotrode models have been designed for rotary ultrasonic welding applications with working frequency of $20 \mathrm{kHz}$ (see Fig. 3a-d).

Sonotrode geometry can be optimized with regard to different factors. Firstly, it could be taken into consideration maximization of the mechanical displacement of the vibration mode on the output surface $A 2$ perimeter in the axis (see Fig. $3 \mathrm{a}-\mathrm{c}$ ) or the radial directions (see Fig. 3d). Secondly, sonotrodes could work in various vibration modes. The sonotrode $\mathrm{S} 1$ has been designed to work in an axial vibration mode. This is the most common case, but not productive one, because of inefficient mechanical displacement distribution concentrated in the middle part of the sonotrode. On the other hand, the sonotrodes S2 and S3 work in the mixed mode of axial and radial vibrations where the displacement amplitude of axial (mostly) and radial vibrations are concentrated on the perimeter, increasing the $P_{\text {ac }}$ energy on the

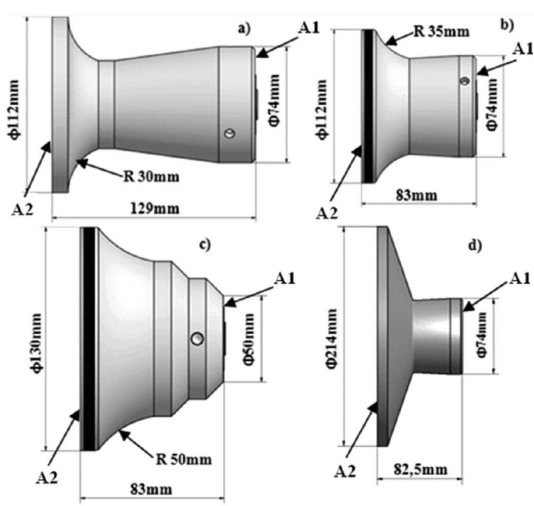

Fig. 3. Mechanical models of developed sonotrodes.

working surface. The sonotrode $S 4$ geometry has been designed to transform input axial vibrations to radial vibrations of the sonotrode ending plate (see Fig. 3).

The proposed sonotrode geometry is composed of a conical shape ended with a plate, which are connected with radius $R$ (see Fig. 3). The conical shape has been applied to concentrate mechanical axis displacement amplitude whereas radius $R$ is responsible for spreading mechanical vibrations on the plate's working surface. The geometry of the $S 4$ sonotrode has been assembled by crossing between two conical shapes, from the input surface $A 1$ to the output surface $A 2$. A rapid crossing has been applied to transform the input axial vibrations into the radial ones.

There are a few problems in the design process that are worth mentioning:

- Rapid transition from a small to a large cross-section (like for designed sonotrodes) results in noticeable changes of mechanical wave distribution, where the plane wave approximation cannot be applied;

- Varied cross-sectional area changes the normal mode vibration frequency;

- Larger radial dimensions of the resonator increase the contribution of the radial modes.

The considered problems lead to such conclusions:

- In order to avoid undesirable radial vibration modes, input diameter of $A 1$ surface cannot be too large compared to the sonotrode axial length,

- The radius $R$ and the generatrix of the used cone geometries need to be optimized to gain the best mechanical displacement and stress distribution with largest output surface diameter for the given frequency.

At the end of this paragraph some practical notes should be mentioned. Firstly, for $20 \mathrm{kHz}$ application input diameter should not exceed $70-80 \mathrm{~mm}$. It was 
found that $74 \mathrm{~mm}$ was still acceptable (see Fig. 3). Secondly, the best mechanical amplitude and velocity transformer have exponential shape, but for small cross-section change it can be successfully replaced by conical shape.

A duraluminum alloy for sonotrode material has been chosen with regard to high mechanical quality factor.

\section{Simulation results}

Conducted simulations were based on several assumptions. Firstly, sonotrode material is isotropic, with density equal to $\rho=2760 \mathrm{~kg} / \mathrm{m}^{3}$, Young modulus $E=$ $72 \mathrm{GPa}$ and Poisson's ratio $=0.33$. Secondly, there are no energy losses due to hysteretic and structural or viscous damping. Thirdly, mechanical constraints are uniformly attached to the sonotrode input surface. Finally, the resonant frequency of all the waveguides has to be tuned to $20 \mathrm{kHz}$. For simulation purpose the MSC FEA 2007 workbench software has been used.

For the purpose of simulation FEM models have been applied which were simplified to $2 \mathrm{D}$ half section models due to the sonotrodes axial isotropy (see Fig. 4).

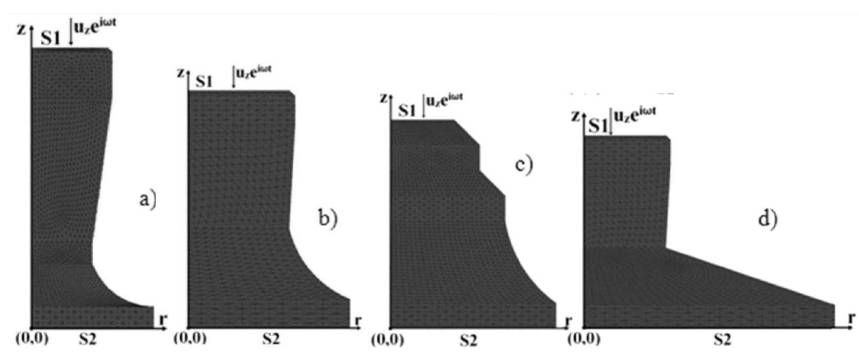

Fig. 4. FEM models of developed sonotrodes.

The distribution of the mechanical displacement amplitude along output $A 2$ surface radius has been optimized in the simulation. To achieve the best welding performance, the highest amplitude has been concentrated at the perimeter of the $A 2$ surface and the lowest one in the middle of the surface. The derived mechanical displacement distribution has been normalized to input mechanical displacement constraint amplitude $u_{z_{\text {in }}}$, which was ascribed value 1 . In Fig. $5 k_{\text {ua }}$ parameter is an amplification factor for displacement amplitude amplification, which can be noted as

$$
k_{\text {ua }}=\frac{u_{\text {out }}}{u_{z \text { in }}}=u_{\text {out }},
$$

where $u_{\text {out }}$ is a displacement amplitude of axial, radial or their composition vibration modes.

TABLE

Sonotrodes normal modes frequencies derived from FEM simulations.

\begin{tabular}{c|c|c|c|c}
\hline \hline Sonotrode & $S 1$ & $S 2$ & $S 3$ & $S 4$ \\
\hline $\begin{array}{c}\text { normal modes } \\
\text { frequency }\end{array}$ & $19945 \mathrm{~Hz}$ & $20162 \mathrm{~Hz}$ & $20014 \mathrm{~Hz}$ & $20085 \mathrm{~Hz}$
\end{tabular}
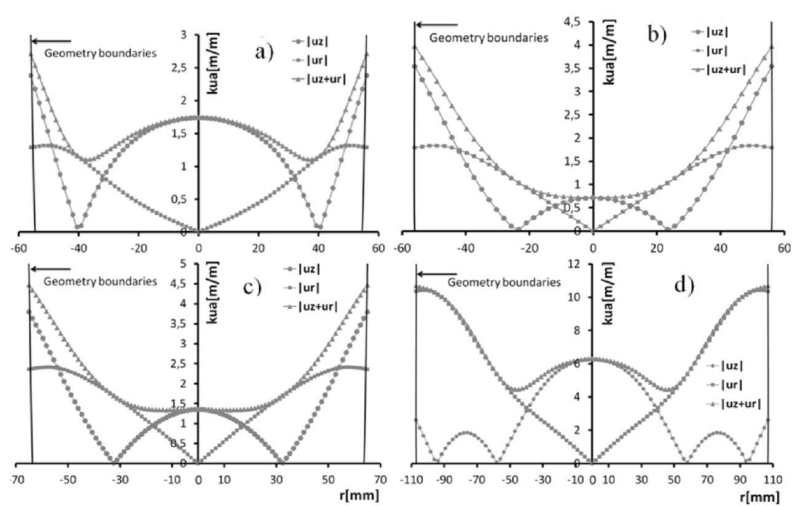

Fig. 5. Mechanical amplitude displacement distribution of axial $u_{z}$ and radial $u_{\mathrm{r}}$ vibration modes along $A 2$ surface output radius, resulted from FEM simulations for sonotrodes, (a) $S 1$, (b) $S 2$, (c) $S 3$, (d) $S 4$.

The simulation results have shown that sonotrode $S 2$ geometry achieves better mechanical displacement distribution compared to other sonotrodes (especially $S 1$ ). Only small quantity of mechanical vibrations is concentrated in the middle part in comparison to outside perimeter of the output surface $A 2$ (see Fig. 5). Simulation has revealed that for $S 2$ and $S 3$ sonotrode geometries mechanical vibrations are highly concentrated on the perimeter of the sonotrodes with good amplification factor (around 4, see Fig. 5). For $S 4$ sonotrode, amplification of the radial mode vibration on the output working surface perimeter is almost 5 times greater than axial mode vibration with high amplitude radial displacement amplitude amplification factor (around 10, see Fig. 5). For every simulated sonotrodes $20 \mathrm{kHz}$ normal frequency has been obtained, which has been presented in Table.

\section{Measurements}

To validate simulation results the electrical impedance characteristic and mechanical displacement measurements of the ultrasonic stack have been carried out.

The measured ultrasonic stack has been composed of high power $(3 \mathrm{~kW})$ sandwich transducer, booster with amplification ratio equal to 1.5 and sonotrode $S 2$. All measurements have been carried out in unloaded conditions, i.e. with the ultrasonic stack mounted in a holder and freely vibrating in the air medium.

Measured impedance characteristics have shown that series and parallel resonant frequency of the ultrasonic stack is near $20 \mathrm{kHz}$. The distance between series and parallel resonant frequencies is in range of $30 \mathrm{~Hz}$ with impedance in series resonance of $\approx 50 \Omega$ (see Fig. 6).

Connected waveguides narrow the resonant (series and parallel) frequencies and mechanically load the converter, as well as increase the electrical load. Frequency range of $30 \mathrm{~Hz}$ is acceptable for ultrasonic stack consisting of converter, booster and sonotrode and can be electrically excited to work. Electrical impedance (measured in unloaded condition) is mostly influenced by energy losses 


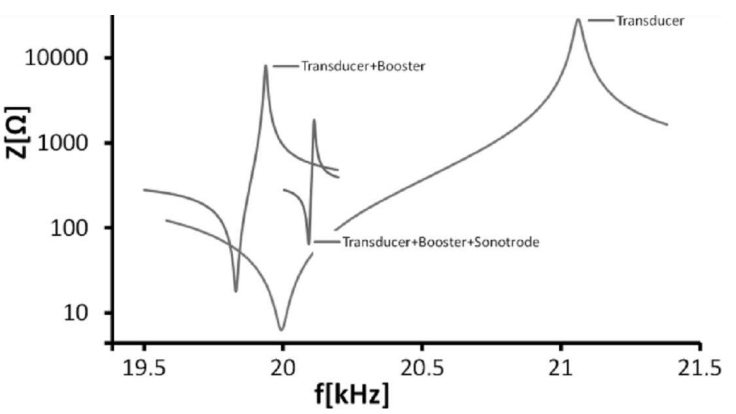

Fig. 6. Ultrasonic vibration system impedance characteristics interfaced with $S 2$ sonotrode.

such as internal friction, acoustic wave radiation to air and dielectric losses in piezoceramics of the converter and others, which are undesirable.

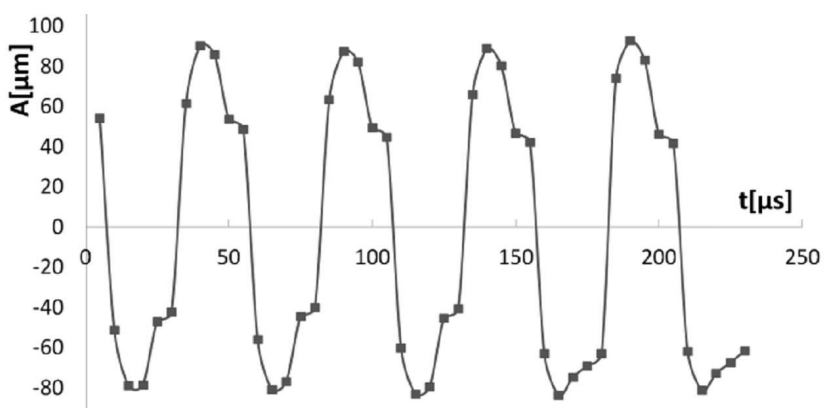

Fig. 7. Axial displacement measurements of $S 2$ sonotrode, measured on $A 2$ surface perimeter, for $300 \mathrm{~W}$ of transducer electrical input power.

Ultrasonic stack has been supplied with $300 \mathrm{~W}$ of electrical power, of harmonic signal with frequency equal to series resonant frequency of the stack. During free vibration of the stack, axial displacement has been measured with laser rangefinder. Displacement has been measured parallel to output surface $A 2$, normal vector towards the perimeter. Results of the measurement have been presented in Fig. 7. The measured amplitude of the axial displacement was in range of $100 \mu \mathrm{m}$, achieving very high value (typical displacement amplitude in many welding processes is in range of $10 \mu \mathrm{m}$ to $50 \mu \mathrm{m}$ ).

\section{Conclusions}

Designed sonotrodes have very promising parameters for application in rotary ultrasonic welding systems. Simulation have shown that in the new sonotrodes mechanical vibrations are distributed on the perimeter, and their high concentration on the working surface have been achieved. In future simulations will be carried out that will include acoustic losses which can have some influence on the mechanical displacement amplitude and thermal effects on junction surface of the welded materials.

\section{Acknowledgments}

We gratefully acknowledge the financial support of the National Science Centre, Poland, under grant number N N510 601840.

\section{References}

[1] M.Đ. Radmanović, D.D. Mančić, Designing and Modeling of the Power Ultrasonic Transducers, MPI, Switzerland 2004.

[2] A. Milewski, P. Kluk, P. Kogut, J. FlorkowskaTrąbińska, Elektronika 52, 194 (2011) (in Polish).

[3] A. Milewski, P. Kogut, P. Kluk, W. Kardyś, Elektronika 53, 15 (2012) (in Polish).

[4] E. Eisner, J. Acoust. Soc. Am. B 41, 1126 (1967).

[5] P.A. Martin, Acoust. Soc. Am. J. 116, 1381 (2004).

[6] O.C. Zienkiewicz, R.L. Taylor, The Finite Element Method for Solid and Structural Mechanics, 6th ed., Elsevier Ltd., Cambridge 2005. 